\title{
Editor Column
}

\section{Direct Submissions from bioRxiv}

This week, The Journal of Neuroscience has taken the next step in reinforcing our commitment to open science by initiating a direct submission process for manuscripts posted on bioRxiv. With this process, preprints can be submitted for peer review at JNeurosci with one click from the bioRxiv site, allowing a more seamless integration between preprint posting and review at the Journal. We believe that this will help authors who want the benefits of community feedback as well as rigorous peer and editorial input.

The classical peer review process and preprint sharing are complementary processes that can and should exist side by side. At JNeurosci, we have supported posting on preprint servers for many years and have an explicit policy that this does not count as previous publication for articles submitted to the Journal (http://www.jneurosci.org/content/general-information\#policies).
The Journal of Neuroscience is committed to a number of essential principles, including rigorous and fair peer review and rapid dissemination of scientific results. There has been increasing discussion of these issues in the scientific community, with the Open Science movement advocating for rapid dissemination through circulation of articles on preprint servers before or during (or in lieu of) the classical peer review process. In addition, starting in November 2016, accepted articles are posted online almost immediately to disseminate peer-reviewed findings as quickly as possible.

Please e-mail us with any questions or suggestions at JN_EIC@sfn.org or tweet us at@MarinaP63.

(D) Marina Picciotto, EiC, JNeurosci

DOI:10.1523/JNEUROSCI.3812-16.2016 